\title{
Play Type Recognition in Real-World Football Video
}

\author{
Sheng Chen, Zhongyuan Feng, Qingkai Lu, Behrooz Mahasseni, Trevor Fiez \\ and Alan Fern, Sinisa Todorovic \\ Oregon State University
}

\begin{abstract}
This paper presents a vision system for recognizing the sequence of plays in amateur videos of American football games (e.g. offense, defense, kickoff, punt, etc). The system is aimed at reducing user effort in annotating football videos, which are posted on a web service used by over 13,000 high school, college, and professional football teams. Recognizing football plays is particularly challenging in the context of such a web service, due to the huge variations across videos, in terms of camera viewpoint, motion, distance from the field, as well as amateur camerawork quality, and lighting conditions, among other factors. Given a sequence of videos, where each shows a particular play of a football game, we first run noisy play-level detectors on every video. Then, we integrate responses of the play-level detectors with global game-level reasoning which accounts for statistical knowledge about football games. Our empirical results on more than 1450 videos from 10 diverse football games show that our approach is quite effective, and close to being usable in a real-world setting.
\end{abstract}

\section{Introduction}

A major part of game planning for American football teams is the collection, annotation, and analysis of game video of their own and opponent games. A number of companies offer web services for facilitating this video-based game planning. However, significant human labor is still involved in using the services, since they usually provide only basic user interface functionalities for manual organization, annotation, and sharing of video. For example, video annotation for just a single game requires entering data for an average of around 150 plays. Thus, there is a growing demand for automating at least part of the annotation process.

In this paper, we consider the problem of automatically annotating videos of plays comprising a football game by one of five high-level play types. This problem is be- yond the capabilities of off-the-shelf computer vision tools, largely due to the huge diversity of football videos. The videos have large variance in viewing angles/distances, shot quality, weather/lighting conditions, the color/sizes of field logos/markings, and the scene around the field, ranging from crowds, to players on the bench, to construction equipment. The videos are typically captured by amateurs, and exhibit motion blur, camera jitter, and large camera motion. Further, there is large variation in team strategies, formations, and uniforms. All this makes video rectification, frame-references, and background detection rather challenging, which are critical for existing approaches.

Our primary goal is achieving robustness to such wide variability, while also maintaining a reasonable runtime. To this end, we exploit knowledge about the structure of football games and individual plays. Specifically, we first process each video using noisy detectors for different play types, designed using domain knowledge. For the sequence of videos from a game, the noisy detector outputs are processed by a Hidden Markov Model (HMM), which encodes knowledge about the temporal structure of football games to improve accuracy. To the best of our knowledge this is the first computer vision system that addresses such fully automated annotation of full football games for such a large diversity of football videos.

We conduct this study in close collaboration with one of the largest companies providing the web service for football video having a client base of over 13,000 high school, college, and professional teams. Our results on a set of 10 diverse, real-world football games, comprising over 1450 play videos, show that our approach is effective, and close to being deployable in the near future.

\section{Background and Problem Statement}

Football Video. American football video is organized around the concept of football plays. Each game involves a sequence of approximately $150+$ plays, each lasting approximately 10 to 30 seconds, separated by short time inter- 
vals where no game action occurs, and the teams regroup. Videos are generally captured with a PTZ camera, showing a sideline view of the football field from an elevated location along the sideline (e.g. Figures 1 and 2). A standard video acquisition of a game involves recording a sequence of videos, one for each play in the game, which can then be automatically organized in temporal order.

Play Types. Each play has a distinct type defined by the standard taxonomy. In this paper, we focus on the five highest-level types in the taxonomy. Below, we describe these play types relative to the annotating team, denoted by TeamA. The two most common play types are when TeamA is on Offense $(O)$ or Defense $(D)$, where TeamA is either trying to move the ball forward $(\mathrm{O})$, or prevent the other team from moving the ball forward (D). Each such play starts with both teams lined-up facing each other at the line of scrimmage - the line parallel to the field lines where the ball is located. The play starts when the ball is "snapped" (or passed) from a player called the center to a player called the quarterback, and then both teams begin moving and executing their chosen strategies until the play terminates.

Plays that are not of type $\mathrm{O}$ or D are called special teams plays and there are three major types. In Kick Off $(K)$ plays, one team lines up and kicks the ball down the field to the receiving team. In Punting $(P)$ plays, the offensive team drop-kicks/punts the ball down the field to the opponent. In Field Goal (F) plays, the ball is kicked at the goal posts in order to score points. The number of special teams plays is generally small compared to the number of $\mathrm{O}$ and $\mathrm{D}$ plays.

The Problem. Our input is a sequence of temporally ordered videos comprising all plays from a football game. The desired output is an accurate labeling of each play by one of the five play types.

\section{Related Work}

There are a number of existing approaches for analyzing football videos. However, it is unlikely that they would be successful across our videos, due to the restrictive assumptions made by these approaches, critical for their foreground-background and camera-motion estimation, rectification of the football field, and extraction of relevant video features.

For example, the approaches presented in [2, 12, 10] perform foreground-background estimation, yard-line detection, and camera-motion estimation. These approaches require high-resolution videos, a fixed scale at which the players appear in the video, and prior knowledge of the field model. Consequently, these approaches cannot be used for our videos. Also, approaches that address videos acquired by a moving camera (e.g., [17]) typically make the limiting assumption that a majority of frame pixels fall on the static background football field. This assumption does not hold in our setting, due to our large number of close-up shots and videos with moving audience in the background. Further, methods that deal with $3 \mathrm{D}$ registration of a visible part of the field [5, 4, 3] make the assumptions that the videos are taken under fairly uniform conditions - namely, on the same or very similar football field (or hockey rink), and from the same camera viewpoint and distance - and that outlier pixels belong to moving players, audience, or referees. Therefore, they cannot be applied in our setting.

Regarding feature extraction, most approaches to playtype recognition require accurate clip stabilization and registration to the football field in order to successfully extract video features relevant for recognition - namely, tracks of football players $[15,9,11]$, or histograms of spatiotemporal interest points [14]. However, previous work has demonstrated that successful tracking of football players under accurate video registration is still notoriously difficult $[8,6]$. Recent work seeks to relax the mentioned requirements for feature extraction $[1,16]$, however they rely on several crucial assumptions that do not reliably hold in our web-service setting. A key part of their approach is video registration and background subtraction. This is an expensive process, and either requires the availability of an overhead view of the football field or user interaction to register a video frame of each play. Our experience with similar approaches for registration and background subtraction has shown that they are not very robust in cases of amateur camera-persons and wide variations of viewpoints.

After extracting relevant video features, existing methods typically employ probabilistic generative models for play-type recognition, including a Bayesian network [9], non-stationary Hidden Markov Model [15], topic model [16], and mixture of pictorial-structure model [7]. These models are typically used for each video in isolation. The recent work of [1] has a limited scope, since they make the assumption that the plays being analyzed are all offensive plays. Remarkably, the reported accuracies of the above approaches are often not high, despite their restrictive settings, indicating fundamental challenges.

To our knowledge, our work is the first to consider parsing entire football games by accounting for constraints between consecutive plays in the game, arising from the standard rules of American football. In addition, no previous work has considered such a large variability of football videos that we deal with. This variability negates the aforementioned assumptions made by prior work.

\section{Overview of Our Approach}

Given a sequence of play videos from a football game, we infer play types in three stages: 1) Partial Rectification (Section 5), where field lines are extracted, providing a partial frame of reference for the football field, 2) Play-level recognition (Sections 6-8), where noisy play-type detectors are run for a subset of the play types, and 3) Game-level 
reasoning (Section 9), where a temporal model of football games is used to reason about the noisy detections across the full sequence to provide more accurate labels.

Ideally, we would like to have play-level detectors for each of the play types: O, D, K, F, P. However, our preliminary analysis and experimentation revealed that distinguishing $\mathrm{F}$ (field goals) from $\mathrm{O}$ and $\mathrm{D}$ would be extremely difficult, due to the high visual similarity of these types. Thus, we do not provide a play-level detector for F plays, but rather rely on the game-level analysis for their inference. We do provide the detectors for the remaining types.

Our OD-detector (Section 6) classifies a video as either $\mathrm{O}$ or D. For non-OD plays, the output will be arbitrary, either O or D. Our K-detector (Section 7) detects kickoffs. Finally, we provide a non-P-detector (Section 8) that attempts to predict with high precision when a video does not show type P. Our preliminary study revealed that achieving both high precisions and recall in predicting $\mathrm{P}$ would be quite difficult due to its high similarity with certain $\mathrm{O}$ and $\mathrm{D}$ plays. However, achieving high precision on predicting non-P is feasible, and useful for game-level analysis.

\section{Extraction of Field Lines}

As reviewed in Section 3, related work relies heavily on video rectification to help deal with camera motion and background subtraction. Such rectification for our webservice videos appears to be beyond the state-of-the-art. Nevertheless, a partial video rectification based on extracting the field lines can be robustly computed, and still be used for camera-motion and background estimation. The field lines (a.k.a. yard lines) are long, white, parallel lines that repeat every 5 yards along the 100 yards between endzones of the football field. The extraction of yard lines provides a partial rectification in the sense that it does not identify a complete homography from frames to field coordinates. Rather, it allows verifying if a line passing through two locations in a frame is parallel to the field lines. This information is used by our detectors in two ways. First, at the beginning of plays and during some special team plays (e.g., kickoffs), the players tend to be aligned with the yard lines. Second, by observing how video features move relative to extracted yard lines, we can get a rough estimate of camera and foreground motion.

We extract the field lines by detecting and tracking the lines in the Hough space. Given a video, we first extract the field lines in the first frame, and then track them along with book-keeping of newly appearing and disappearing lines in the field-of-view.

Field line extraction: For each video frame, we first run the Hough transform to get a candidate set of lines which intersect at a point (see Figure 1), since parallel lines are bound to intersect at a vanishing point under the perspective transform of the camera. We can then esti-
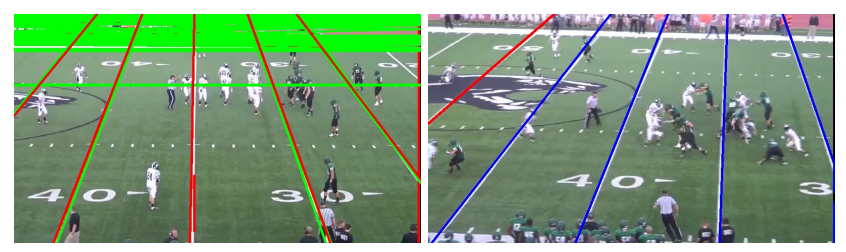

Figure 1. (Left) Line extraction: Green lines are the output from the Hough transform, red lines are the results from RANSAC. (Right) Line tracking: Blue lines are the tracked lines from last frame, the red line is the new line detected in this frame.

mate the Hough parameters of the field lines, $(\rho, \theta)$, as $\rho=x_{0} \cos \theta+y_{0} \sin \theta$, where $\left(x_{0}, y_{0}\right)$ is the location of the vanishing point. This estimation problem can be solved using RANSAC and least squares.

Line tracking: We want to associate extracted lines from the previous and current frames. For this, we conduct meanshift in the Hough space. Since the lines typically do not move much from one frame to another, meanshift converges after 1-2 iterations. To deal with missing and newly appearing lines caused by the camera motion, we perform the following post-processing. For missing lines, the tracked results will be noisy, and thus can be filtered out by RANSAC. Newly arising peaks in the Hough space, which do not correspond to previous lines, are added to the field-line set if they are consistent with the vanishing point model. Figure 1 illustrates our tracking of the field lines.

\section{O-D Detector}

Each O or D play begins at the moment-of-snap (MOS) with the two teams on opposite sides of the line-ofscrimmage (LOS) (see Figure 2) in either an offensive or defensive formations. Therefore, our O-D detection, first, identifies the MOS and the LOS, and then performs two predictions: i) which side of the LOS (left or right) the offensive team is lined up on at the MOS - called Offensive Direction Inference; and ii) which side of the LOS TeamA is lined up on at the MOS - called Team Direction Inference. Note that in our experiments for each game, we arbitrarily chose one of the teams to be TeamA.

The frame number of the MOS is highly variable across videos, depending on when recording begins. Detecting the MOS is challenging due to unpredictable camera motion and non-player foreground motion. A recent approach to this problem [1] relied heavily on having rectified video and foreground extraction, which is not an option for our setting. Rather, we use another recent approach [13] that does not have such requirements. In [13], a sophisticated analysis of optical flow was used to achieve relatively good MOS estimates on average. Across the $1450+$ videos in our experiments the resulting MOS estimates are within 5 frames of the true MOS for $54 \%$ of the videos, within 15 frames for $85 \%$ of the videos, and within 30 frames for $89 \%$. This level of accuracy allows for non-trivial O-D detection. 

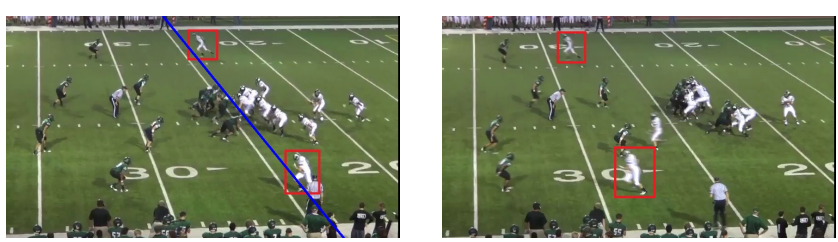

Figure 2. Two frames showing two wide receivers (in red box) running from right to left. The detected LOS is shown as as blue lines in the left image

Offensive Direction Inference. The $\mathrm{O}$ and $\mathrm{D}$ play types differ in the spatial formations of players on each team at the start of the play. However, analyzing these formations requires a reliable player detector. For example, recent work [1], detects the foreground players via video registration and a background model for such analysis, which is unreliable across our videos. Rather than analyzing the entire formation, we instead seek to detect offensive players called, wide receivers (WRs), whose motion is predictive of which side of the LOS the offense is on. As shown in Fig. 2, WRs are players that usually line up at the ends of the field, and are isolated from the other offensive players. A majority of videos of O-D plays show at least one WR. After a play starts, the WRs will almost always immediately run in the direction of the defense. Thus, our approach to offensive direction inference is to detect the initial motion of one or more WRs, and infer that the offense is on the side opposite to the direction of the motion. Note that there are rare cases of O-D plays where there are no WRs on the field. In these cases, our approach will produce arbitrary results.

For detecting WRs, we extract and analyze KLT tracks in order to infer which ones are likely due to WRs, and then infer the WR motion direction based on those trajectories. We run the KLT tracker on a sequence of 45 video frames following the estimated MOS. The extracted point trajectories typically correspond to player movement, non-player foreground motion, and background features under camera motion. When a WR is visible, the KLT tracker is capable of extracting relatively long trajectories due to the WR's characteristic swift and straight-line motion. To help remove KLT trajectories caused by camera motion, we use the extracted field lines (Section 5), by measuring the relative motion of each KLT track to its closest field line. Ideally, this relative motion is small when a KLT track corresponds to a stationary background feature. This allows removing all KLT tracks whose relative motion falls below a threshold. The specific threshold choice is not critical, since the KLT tracks of WRs generally have very large relative motion.

Given the remaining KLT trajectories, the key to inferring which ones belong to WRs is to use our knowledge that: i) WRs are typically located at the far ends of the LOS, and ii) WRs are usually isolated from the majority of players lined up at the LOS. This requires first inferring the LOS. For LOS detection, we draw on an idea from [1] that

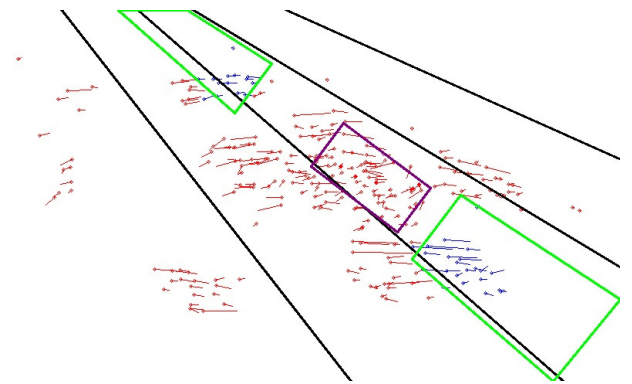

Figure 3. Plot of one video's KLT tracks with detected scrimmage line (purple box) and ranges for WRs (green boxes). Red (blue) vectors are the KLT tracks outside (inside) the range of the WRs.

the LOS is usually in the region of highest image gradients corresponding to the "texture" of players on the "uniform" field. Further, we know that the LOS is always parallel to the field lines. We use a sliding window to scan across the frame for the region of highest gradients, where the medial axis of the window is enforced to pass through the estimated vanishing point of the field lines (i.e., to be "parallel" under perspective to the field lines). As we scan, the window size is adapted to the distance between the detected field lines.

The sliding window with maximum gradient gives a good estimate of the location of the offensive and defensive lines of players. We use two other windows on each side of this maximum-gradient window to identify likely areas where WRs are located. Figure 3 shows an example of extracted KLT tracks, with the maximum-gradient window, and the two WR windows.

Finally, we estimate the motion direction of WRs using a weighted average of the KLT trajectories in the two WR windows. In particular, we linearly weight each KLT track according to its distance from the center of the maximumgradient window. This assigns higher weights to KLT tracks that are more isolated, and hence more likely to be due to WR's motion. We use the direction of the vector resulting from the weighted average, relative to the field lines, as the estimated direction of the WR. Note that in cases where either the upper or lower WR is not present in the video, this combination will tend to be biased in favor of the visible WR, since there will typically be few highly weighted KLT tracks in the WR window not containing a WR.

Team Direction Inference. A football game consists of a sequence of plays and can be further grouped into four quarters. Throughout each quarter, each team will always face the same direction, and the directions are switched after the first and third quarters. The directions of the teams at the start of each half (i.e., the first and third quarters) are somewhat arbitrary and decided by coin tosses. Based on this description, we see that games will have long sequences of plays where the team direction does not change. The key to inferring the team direction of plays is to detect the split points where the team directions change (there is 
a maximum of 3 split points). One piece of additional information that is useful for this analysis is the video time stamps, which are used by the web-service to temporally order videos. In particular, between the second and third quarters, there is a prolonged half-time break that is easily detectable as the maximum duration gap between plays in a game. Thus, we can easily determine the starting video of the third quarter based on the time stamps.

We detect the split point $s_{1}$ in the first half between the first and second quarters, and the split point $s_{2}$ in the second half between the third and fourth quarters, where we know that the team directions must have changed. Given $s_{1}$ and $s_{2}$, we then estimate if there was a change in direction between the two halves, which uniquely identifies the second and third quarter. Intuitively, at a true split point we should notice the change in color profile of the teams to the left and right of the LOS, since the teams wear different uniforms. Thus, in the first half we evaluate each possible value of $s_{1}$ (correspondingly $s_{2}$ in the second half) by the color differences between plays, before and after $s_{1}$, as follows:

$$
\operatorname{Score}\left(s_{1}\right)=\sum_{\substack{1 \leq j \leq s_{1} \\ s_{1}+1 \leq k \leq N_{1}}} \operatorname{Diff}(j, k)
$$

Here $N_{1}$ is the play number of the final play of the first half and $\operatorname{Diff}(j, k)=d\left(j_{l}, k_{r}\right)+d\left(j_{r}, k_{l}\right)$, where $d()$ is the Euclidean distance, and $j_{l}, j_{r}$ are the color histograms to the left and right of the LOS for play $j$, respectively. To compute the color histograms, we consider pixels to the left and right of the LOS within the maximum-gradient window described above. Using Score $\left(s_{1}\right)$ we pick the top scoring split point $s_{1}$ as our estimate. Similarly we infer that the directions between the second and third quarters change if the corresponding score is above a threshold, or otherwise that the direction did not change. This gives us the direction estimation for TeamA across the entire game.

\section{Kickoff Detector}

Kickoff (K) plays occur at the beginning of the game, the beginning of the second half of the game, and after any team scores, when the scoring team kicks the ball off. The most visually distinct aspect of $\mathrm{K}$ plays is the line of players on the kicking team lining up at one end of the field, usually spanning the entire width of the field, and running down the field to tackle whoever catches the ball on the other team. Figure 4 (left) shows a frame at the start of a kickoff video, just before the ball is kicked. Players along this line run at approximately the same speed (full speed), and are relatively evenly distributed along the width of the field.

Our K detector: i) identifies the initial time interval of the $\mathrm{K}$ play where the moving player line is most prominent; ii) computes mid-level features about this time interval; and iii) uses the mid-level features in Logistic regression for predicting whether the play is a kickoff or not, where Logis-

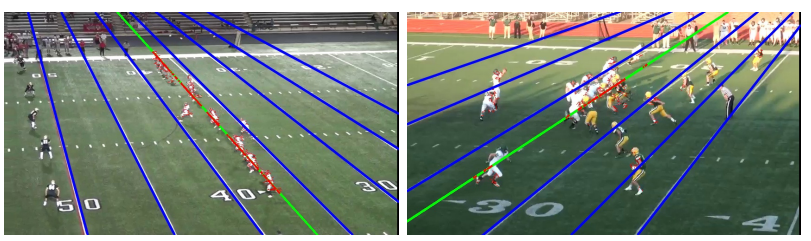

Figure 4. Line fitting results in kickoff (left) and non-kickoff videos (right) depicting foreground KLT tracks (red) and fitted lines (green).

tic regression is learned on training videos of $\mathrm{K}$ and non- $\mathrm{K}$ plays. Below, we explain these steps.

Identifying Initial Time Interval of the K Play. We found that the MOS detector used for O-D detection is not sufficiently reliable at detecting the start of kickoff plays, in part, because kickoff videos have much larger variations in camera motion. To detect the start of $\mathrm{K}$ plays, we first estimate frame-to-frame homographies aimed at reducing the impact of camera motion. The homographies are estimated by RANSAC over Harris-corner pairs matched in consecutive frames. The matched Harris corners are likely to belong to the background. Then, we run the KLT tracker only on the unmatched Harris corners which are likely to belong to the foreground players. We monitor the number of such foreground KLT tracks, and when a threshold is exceeded we flag the start of the $\mathrm{K}$ play. While this homography estimation is noisy, and not robust for long-range registration, it is sufficiently accurate for the purposes of identifying foreground KLT tracks. We use a fixed length time interval of 2 seconds after the estimated start of the K play.

Kickoff Features. Given the above time interval, we compute mid-level features related to the moving line of players. In each frame of the time interval, we use RANSAC to fit a line to the start points of the foreground KLT tracks. Line fitting is done in the Hough space, and constrained so that the line slope is close (under perspective) to that the closest extracted yard line, since the player line is generally parallel to the yard lines. Fig. 4 shows our line fitting in frames from kickoff and non-kickoff videos.

The mid-level features measure properties of the player lines fitted in the sequence of frames, including: 1) The entropy of the spatial-layout distribution of the foreground KLT tracks along the extracted line. The spread distribution is usually uniform for K plays, yielding high entropy. 2) The distance that the fitted line of the foreground KLT tracks covers during the initial time interval relative to the field lines. For $\mathrm{K}$ plays this relative distance will typically be much larger than in non-kickoff plays. 3) Two measures of team movement consistency, which indicates how much the fitted lines across the sequence of frames agree with each other in terms of their slope and the movement direction. 


\section{Non-Punt Detection}

In punting plays, both teams line up and initially behave as in O-D plays. However, at the start of the P play, the punter kicks the ball deep down the field, and the kicking team runs down the field to tackle the opposing player that catches the ball. This makes $\mathrm{P}$ plays quite similar to $\mathrm{O}$, $\mathrm{D}$, and $\mathrm{K}$ types. Thus, designing a robust $\mathrm{P}$ detector is challenging. However, for our game-level analysis, it is still quite useful to have a detector that can reliably indicate when a play is not a punt. That is, we here aim for a high precision detector for non-punt plays, possibly at the expense of recall.

Our non-punt (non-P) detector is based on two characteristics of P plays. First, since the ball is kicked far down the field, the camera usually pans fairly rapidly in order to follow the action. Such rapid panning near the beginning of a P play is not common for O-D plays. Second, a short time after the $P$ play begins, most of the players on the kicking team will run down the field, which is uncommon at the start of O-D plays. We measure these characteristics using the methods from the above kickoff detector.

In particular, we estimate homographies between consecutive frames during a period after the MOS, in order to detect a significant burst in camera motion. For this, we looking for sequences of consistently large changes in the homographies. Next, we also use the lines fit by the kickoff detector to look for significant team movement down field in a short period of time. Our final non-P detection is obtained by thresholding the camera movement and team movement cues. If neither threshold is exceeded we output that the play is not a punt, since P plays should exceed at least one of the thresholds. The thresholds were tuned using training data of punts and non-punts in order to optimize the precision without sacrificing recall too much.

\section{Game Level Prediction}

The input to our game-level analysis is the sequence of $\mathrm{O}, \mathrm{D}, \mathrm{K}$, and non-P detections for each play in a game. The goal of the game-level analysis is to help correct for detection errors and introduce $\mathrm{F}$ labels by taking into account the statistical regularities in sequences of football plays.

The statistical regularities are captured by a Hidden Markov Model (HMM), which models a sequence of observations $O_{1}, \ldots, O_{T}$ as being generated by a sequence of hidden states $S_{1}, \ldots, S_{T}$. In our case, the hidden state $S_{t}$ is the play type of the $t$ th play, which is either $\mathrm{O}, \mathrm{D}, \mathrm{K}, \mathrm{F}$, $\mathrm{P}$. The observation $O_{t}$ corresponds to the cross-product of the detector outputs. An HMM is specified by a state transition distribution $P\left(S_{t} \mid S_{t-1}\right)$ which gives the probability of transitioning to a particular state $S_{t}$ when the previous state is $S_{t-1}$. For example, the probability of a transition from $S_{t-1}=O$ to $S_{t}=O$ is much higher than a transi-

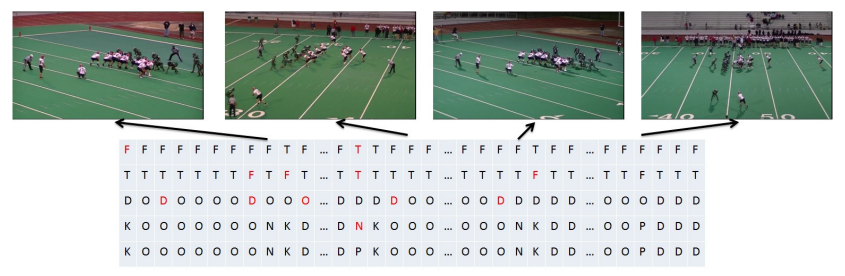

Figure 5. Game-Level Analysis. Row 1: kickoff detector, T is for kickoff and $\mathrm{F}$ for non-kickoff. Row 2: non-punt detector, $\mathrm{T}$ for non-punt and F for punt. Row 3: OD detector, Row 4: Viterbi output, Row 5: ground truth. Errors are shown in red.

tion to $S_{t}=D$. The HMM also specifies an observation distribution $P\left(O_{t} \mid S_{t}\right)$ which gives the probability of observing observation $O_{t}$ when the hidden state is $S_{t}$. For example, for the state $S_{t}=O$ the most likely observation corresponds to the OD detector predicting $\mathrm{O}$, the $\mathrm{K}$ detector predicting false, and the non-punt detector predicting true.

The HMM transition and observation probabilities are estimated as the Laplace-smoothed Maximum Likelihood Estimates using hand-labeled training games.

Given the transition and observation distributions, it is straightforward to use the Bayes rule, and compose them into a conditional distribution, $P\left(S_{1}, \ldots, S_{T} \mid O_{1}, \ldots, O_{T}\right)$, which gives the probability of a state sequence given an observation sequence. Thus, given an observation sequence from a game, we find the state sequence that maximizes the above conditional probability, and returns the result as the final assignments for the game. Computing the most-likely state sequence can be done using the well-known Viterbi algorithm, which is a polynomial time dynamic programming algorithm.

Figure 5 illustrates the game-level analysis on one of our evaluation games. In this case the analysis is able to correct all detector mistakes except for one, where it predicts $\mathrm{K}$ instead of P. Here at the P play, the receiving team loses the ball and the kicking team directly gets a touchdown which rarely happens. At the same time, the non-P detector made a mistake, which gives us no confidence that this is a $\mathrm{P}$ play. Given just this detector input, most human experts would make the same judgement as our analysis in this situation.

\section{Evaluation}

We performed empirical evaluation on 10 high-school football games, which were selected by the web-service company from their database in an attempt to cover the wide diversity of video. Each game has a sequence of an average of 146 videos. The videos were hand-labeled by their types. We also annotated the true MOS, and team direction in each play to allow for more refined evaluations. Training and threshold selecting for all the detectors was done in a different dataset.

Detector Evaluation. Table 1 shows the results for offense direction inference (WR), team direction inference 


\begin{tabular}{|l|c|c|c|c|c|c|}
\hline Dataset & TD $^{*}$ & WR $^{*}$ & OD* & TD & WR & OD \\
\hline \hline Game01 & 1.00 & 0.97 & 0.97 & 0.94 & 0.83 & 0.80 \\
Game02 & 0.99 & 0.84 & 0.84 & 0.98 & 0.80 & 0.82 \\
Game03 & 1.00 & 0.85 & 0.85 & 0.99 & 0.76 & 0.76 \\
Game04 & 0.89 & 0.79 & 0.71 & 0.91 & 0.62 & 0.62 \\
Game05 & 0.96 & 0.83 & 0.81 & 0.90 & 0.77 & 0.71 \\
Game06 & 0.99 & 0.86 & 0.85 & 0.99 & 0.79 & 0.78 \\
Game07 & 0.90 & 0.66 & 0.63 & 0.90 & 0.63 & 0.63 \\
Game08 & 0.98 & 0.93 & 0.92 & 0.94 & 0.92 & 0.87 \\
Game09 & 0.97 & 0.93 & 0.91 & 0.99 & 0.93 & 0.92 \\
Game10 & 0.97 & 0.89 & 0.87 & 0.97 & 0.82 & 0.80 \\
\hline \hline Overall & 0.97 & 0.85 & 0.84 & 0.95 & 0.79 & 0.77 \\
\hline
\end{tabular}

Table 1. Accuracy for OD detector. TD is for team direction, WR is for offensive direction, and OD is the combined OD detector. $\mathrm{TD}^{*}, \mathrm{WR}^{*}$ and $\mathrm{OD}^{*}$ are results using ground truth MOS.

(TD), and the combined OD detection accuracy. Accuracy is computed only with respect to plays that are truly $\mathrm{O}$ and D. This includes plays where a penalty occurred, which means that there is no player motion and thus our OD detector typically makes errors. Since the OD detector components are dependent on MOS estimation, we show results for the OD detector when using the automatically predicted MOS, and also the ground truth MOS (denoted as WR*, $\mathrm{TD}^{*}$, and $\left.\mathrm{OD}^{*}\right)$. We see that using the predicted MOS the overall OD accuracy is $77 \%$ across all games, which is a significant improvement over random guessing. Using the true MOS improves that accuracy to $84 \%$. From the table, the difference in accuracy between using the predicted and true MOS is largely due to decreased accuracy of the offensive direction estimate (WR vs. WR*). This is because, when the MOS prediction is inaccurate, the KLT tracks analyzed may sometimes not include WR tracks. Thus, further improving the MOS estimation or extending the WR detector to search in a wider temporal window around the predicted MOS are possible ways to improve OD accuracy.

The OD accuracy can vary substantially across games, primarily due to varying quality of camerawork, containing more jitter and unnecessary motion for some games. This indicates that considering efficient ways to more reliably counteract camera motion would be an important direction for improvement.

We use a different dataset of 293 plays including 74 kickoffs to train the parameters of the kickoff detector (ko), and measure the precision and recall across the 10 games, as shown in Table 2.

From the table, we achieve non-trivial precision and recall overall. However, there are some games with relatively low precision (Game08) or recall (Game05). This is mainly caused by the fact that appearance of both the fields and kicking-team uniforms are dominated by dark green, which makes Harris corner extraction less accurate. Another reason for errors is that some OD plays look similar to kickoffs,

\begin{tabular}{|c|c|c|c|c|}
\hline Dataset & Pre $_{k o}$ & Recall $_{k o}$ & Pre $_{n p}$ & Recall $_{n p}$ \\
\hline \hline Game01 & $100.00 \%$ & $88.89 \%$ & $97.30 \%$ & $90.00 \%$ \\
Game02 & $75.00 \%$ & $75.00 \%$ & $100.00 \%$ & $73.33 \%$ \\
Game03 & $100.00 \%$ & $90.00 \%$ & $100.00 \%$ & $78.31 \%$ \\
Game04 & $100.00 \%$ & $83.33 \%$ & $100.00 \%$ & $76.06 \%$ \\
Game05 & $90.00 \%$ & $75.00 \%$ & $96.52 \%$ & $86.72 \%$ \\
Game06 & $80.00 \%$ & $100.00 \%$ & $100.00 \%$ & $83.33 \%$ \\
Game07 & $78.57 \%$ & $100.00 \%$ & $99.17 \%$ & $80.95 \%$ \\
Game08 & $61.54 \%$ & $88.89 \%$ & $96.99 \%$ & $87.76 \%$ \\
Game09 & $100.00 \%$ & $100.00 \%$ & $98.02 \%$ & $83.19 \%$ \\
Game10 & $85.71 \%$ & $85.71 \%$ & $97.56 \%$ & $56.74 \%$ \\
\hline \hline Overall & $85.41 \%$ & $88.17 \%$ & $98.57 \%$ & $79.41 \%$ \\
\hline
\end{tabular}

Table 2. Result of kickoff and non-punt detector. Pre $e_{k o}$ and $\operatorname{Pre}_{n p}$ are the precision for kickoff and non-punt respectively. Recall $_{k o}$ and Recall ne $_{p}$ are the recall.

because both teams rush toward the same direction after the MOS, and are relatively uniformly spread out, as in kickoffs. Table 2 also shows results for the non-punt detector. As expected, we do get high precision. One reason for the low recall is that the detector often mistakes a kickoff play for a punt.

Overall System. Since our HMM requires training of its parameters, we used a leave-one-game-out strategy for evaluation. Thus, we present the average results for each game using an HMM trained on the other 9 games. In addition to evaluating our fully automated system, we also test a version of the system that replaces each detector by the ground truth. This allows us to observe which detectors would be most useful to improve in order to increase overall accuracy. We denote our kickoff, non-punt, MOS and OD detectors as $k o, n p, m o s$ and $o d$, respectively. We use $G T_{d}$ to denote that we use the ground truth for detector $d$. Our fully automated system is denoted as $G T_{\emptyset}$. The system that replaces all detectors by ground truth is denoted as $G T_{\text {all }}$. Table 3 shows the results.

First, we see that the overall performance of our fully automated system $G T_{\emptyset}$ is $77 \%$ compared to the accuracy of $44 \%$ for random guessing according to the play type proportion prior (O, D for around $41 \%, \mathrm{~K}, \mathrm{P}$ for $6 \%, \mathrm{~N}$ for $5 \%$ and $\mathrm{F}$ for $1 \%$ in our dataset). This level of accuracy is in the range of being usable in practice given a proper user interface. The user could change the label of any mislabeled videos they come across. This type of interface could, for example, allow team coaches to analyze the types of plays they are interested more quickly than is currently possible.

From Table 3, even with the ground truth detector input $G T_{\text {all }}$, we still cannot get perfect results. That is, the upper bound of our performance is below $100 \%$ accuracy. The main reason is that we do not have a field goal detector, and the game-level analysis does not perfectly predict where to insert field goal labels. Regarding the impact of each detector, we see that by far using the ground truth OD 


\begin{tabular}{|c|c|c|c|c|c|c|}
\hline Dataset & $G T_{\text {all }}$ & $G T_{\emptyset}$ & $G T_{\text {mos }}$ & $G T_{o d}$ & $G T_{k o}$ & $G T_{n p}$ \\
\hline \hline Game01 & 0.98 & 0.79 & 0.94 & 0.95 & 0.81 & 0.81 \\
Game02 & 0.99 & 0.85 & 0.83 & 0.92 & 0.88 & 0.85 \\
Game03 & 0.98 & 0.74 & 0.78 & 0.94 & 0.72 & 0.82 \\
Game04 & 0.99 & 0.65 & 0.76 & 0.96 & 0.62 & 0.71 \\
Game05 & 0.99 & 0.72 & 0.83 & 0.93 & 0.77 & 0.76 \\
Game06 & 0.99 & 0.82 & 0.85 & 0.96 & 0.82 & 0.90 \\
Game07 & 0.99 & 0.69 & 0.71 & 0.98 & 0.69 & 0.75 \\
Game08 & 0.98 & 0.77 & 0.87 & 0.92 & 0.81 & 0.83 \\
Game09 & 0.98 & 0.84 & 0.89 & 0.96 & 0.84 & 0.93 \\
Game10 & 0.98 & 0.67 & 0.91 & 0.93 & 0.70 & 0.85 \\
\hline \hline Overall & 0.99 & 0.77 & 0.83 & 0.95 & 0.79 & 0.85 \\
\hline
\end{tabular}

Table 3. Accuracy of the overall system

\begin{tabular}{|c|c|c|c|c|c|}
\hline & $\mathrm{O}$ & $\mathrm{D}$ & $\mathrm{K}$ & $\mathrm{F}$ & $\mathrm{P}$ \\
\hline $\mathrm{O}$ & 0.77 & 0.14 & 0.01 & 0.01 & 0.07 \\
\hline $\mathrm{D}$ & 0.13 & 0.80 & 0.01 & 0.02 & 0.04 \\
\hline $\mathrm{K}$ & 0.04 & 0.07 & 0.84 & 0.01 & 0.03 \\
\hline $\mathrm{F}$ & 0.10 & 0.07 & 0.01 & 0.72 & 0.09 \\
\hline $\mathrm{P}$ & 0.27 & 0.25 & 0.02 & 0.01 & 0.44 \\
\hline
\end{tabular}

Table 4. Confusion matrix for $G T_{\emptyset}$. Rows correspond to ground truth labels and columns correspond to predicted labels.

labels has the biggest impact on accuracy. This is largely because the number of OD labels is dominant and there is still significant room to improve our OD detector. We can also achieve an $8 \%$ increase with the ground truth non-punt detector since punts are one of the most common bridges between OD transitions.

The confusion matrix for our automated system is shown in Table 4. We see that the least accurate label is P, which is often predicted as $\mathrm{O}$ or $\mathrm{D}$. This is partially due to the fact that our non-punt detector is biased toward high precision, so that when it predicts false (the play may be a punt) it is still quite likely that the play is a non-punt.

Time Complexity. Our primary focus has not yet turned to time optimization. Nevertheless, we achieve reasonable runtimes. Our code is implemented in $\mathrm{C} / \mathrm{C}++$, and tested on a Red Hat Enterprise 64 bit, $3.40 \mathrm{GHZ}$ environment. For the kickoff detector, the processing speed is $25-30$ frames per second on average. The MOS detector processes 3035 frames per second on average. The offensive and team direction estimates require an average of 25 and $20 \mathrm{sec}-$ onds for a video respectively. The non-punt detector can be viewed as part of the kickoff detector and thus requires no additional time. The game-level analysis also takes practically no time compared with the detectors. Thus, in total, for a video with 600 frames (20s), the average processing time will be 85 seconds. If we run the offensive direction and team direction detector in parallel and the kickoff and OD detector in parallel, the parallel time will be 45 seconds which is approximately twice the video length. In addition, every detector is run independently in our experiment while some components can be shared across detectors such as the KLT tracks and line of scrimmage estimation, so there is still significant room for reducing the running time.

\section{Conclusion}

We have described the first computer vision system for recognizing sequences of plays of entire football games. Our evaluation is based on perhaps the widest variation of video conditions considered in any prior work on football analysis. The evaluation shows that currently we are able to achieve $77 \%$ accuracy on labeling plays as either offense, defense, kickoffs, punting, or field goals, under reasonable running times. In this sense, our system is close to being usable in a real-world web-service setting. We have also identified most promising directions for prospective improvement of the accuracy and efficiency of our system.

\section{References}

[1] I. Atmosukarto, B. Ghanem, S. Ahuja, K. Muthuswamy, and N. Ahuja. Automatic recognition of offensive team formation in american football plays. In CVPR Workshops (CVsports), 2013. 2, 3,4

[2] Y. Ding and G. Fan. Camera view-based american football video analysis. In IEEE ISM, 2006. 2

[3] B. Ghanem, T. Zhang, and N. Ahuja. Robust video registration applied to field-sports video analysis. In ICASSP, 2012. 2

[4] A. Gupta, J. Little, and R. Woodham. Using line and ellipse features for rectification of broadcast hockey video. In Canadian Conference on Computer and Robot Vision (CRV), 2011. 2

[5] R. Hess and A. Fern. Improved video registration using nondistinctive local image features. In CVPR, 2007. 2

[6] R. Hess and A. Fern. Discriminatively trained particle filters for complex multi-object tracking. In $C V P R, 2009.2$

[7] R. Hess, A. Fern, and E. Mortensen. Mixture-of-parts pictorial structures for objects with variable part sets. In ICCV, 2007. 2

[8] S. Intille and A. Bobick. Closed-world tracking. In ICCV, 1995. 2

[9] S. S. Intille and A. F. Bobick. Recognizing planned, multiperson action. Computer Vision and Image Understanding, 81(3):414-445, 2001. 2

[10] B. L. and M. I. Sezan. Event detection and summarization in sports video. In CBAIVL, 2001. 2

[11] R. Li, R. Chellappa, and S. Zhou. Learning multi-modal densities on discriminative temporal interaction manifold for group activity recognition. In $C V P R, 2009.2$

[12] T.-Y. Liu, W.-Y. Ma, and H.-J. Zhang. Effective feature extraction for play detection in american football video. In $M M M, 2005.2$

[13] B. Mahasseni, S. Chen, A. Fern, and S. Todorovic. Detecting the moment of snap in real-world football videos. In IAAI, 2013. 3

[14] B. Siddiquie, Y. Yacoob, and L. S. Davis. Recognizing plays in american football videos. Technical report, University of Maryland, 2009. 2

[15] E. Swears and A. Hoogs. Learning and recognizing complex multiagent activities with applications to american football plays. In WACV, 2012. 2

[16] J. Varadarajan, I. Atmosukarto, S. Ahuja, B. Ghanem, and N. Ahuja. A topic model approach to represent and classify american football plays. In $B M V C, 2013.2$

[17] S. Wu, O. Oreifej, and M. Shah. Action recognition in videos acquired by a moving camera using motion decomposition of lagrangian particle trajectories. In $I C C V, 2011.2$ 